LBNL-39155

UCB-96/35

hep-ph/9607463

July, 1996

\title{
Non-unified gaugino masses in supersymmetric missing partner models with hypercolorf
}

\author{
Nima Arkani-Hamed ${ }^{a, b}$, Hsin-Chia Cheng ${ }^{a, b}$ and Takeo Moroi ${ }^{a}$ \\ ${ }^{a}$ Theoretical Physics Group, Lawrence Berkeley National Laboratory \\ University of California, Berkeley, CA 94720, U.S.A. \\ ${ }^{b}$ Department of Physics, University of California, Berkeley, CA 94720, U.S.A.
}

\begin{abstract}
The gaugino mass relations $m_{3} / g_{3}^{2}=m_{2} / g_{2}^{2}=m_{1} / g_{1}^{2}$ are considered to be robust signals for supersymmetric grand unification. In this letter, we point out that these relations may be significantly modified in an interesting class of models which solve the doublet-triplet splitting problem using a missing partner mechanism together with a strong hypercolor gauge group. The observation of non-unified gaugino masses, together with unified sfermion masses, provides a distinctive signature for these models.
\end{abstract}

${ }^{*}$ This work was supported in part by the Director, Office of Energy Research, Office of High Energy and Nuclear Physics, Division of High Energy Physics of the U.S. Department of Energy under Contract DE-AC03-76SF00098 and in part by the National Science Foundation under grant PHY-95-14797. 


\section{Disclaimer}

This document was prepared as an account of work sponsored by the United States Government. While this document is believed to contain correct information, neither the United States Government nor any agency thereof, nor The Regents of the University of California, nor any of their employees, makes any warranty, express or implied, or assumes any legal liability or responsibility for the accuracy, completeness, or usefulness of any information, apparatus, product, or process disclosed, or represents that its use would not infringe privately owned rights. Reference herein to any specific commercial products process, or service by its trade name, trademark, manufacturer, or otherwise, does not necessarily constitute or imply its endorsement, recommendation, or favoring by the United States Government or any agency thereof, or The Regents of the University of California. The views and opinions of authors expressed herein do not necessarily state or reflect those of the United States Government or any agency thereof of The Regents of the University of California and shall not be used for advertising or product endorsement purposes.

Lawrence Berkeley Laboratory is an equal opportunity employer. 
Supersymmetric grand unified theory (SUSY-GUT) [1] is one of the most attractive candidates of new physics beyond the standard model. It is supported by the precision measurements of the gauge coupling constants of $\mathrm{SU}(3)_{\mathrm{C}} \times \mathrm{SU}(2)_{\mathrm{L}} \times \mathrm{U}(1)_{\mathrm{Y}}$ [2]. However, it may be that the unification of gauge couplings is an accident. What further evidence can support the existence of a SUSY-GUT? If supersymmetric particles are discovered, we may hope to see signatures for unification in the superpartner spectrum. In particular, the pattern of sfermion masses can probe the unification of quark and lepton multiplets, while the gaugino masses can signal the existence of a unified gauge group at the GUT scale. Between the two, the weak scale scalar mass relations are more sensitive to the physics between the weak and GUT scales, whereas the gaugino mass relations

$$
\frac{m_{3}}{g_{3}^{2}}: \frac{m_{2}}{g_{2}^{2}}: \frac{m_{1}}{g_{1}^{2}}=\frac{m_{\mathrm{GUT}}}{g_{\mathrm{GUT}}^{2}}: \frac{m_{\mathrm{GUT}}}{g_{\mathrm{GUT}}^{2}}: \frac{m_{\mathrm{GUT}}}{g_{\mathrm{GUT}}^{2}}=1: 1: 1,
$$

where $g_{3}, g_{2}, g_{1}$ and $g_{\mathrm{GUT}}\left(m_{3}, m_{2}, m_{1}\right.$ and $\left.m_{\mathrm{GUT}}\right)$ represent the gauge coupling constants (gaugino masses) for $\mathrm{SU}(3)_{\mathrm{C}}, \mathrm{SU}(2)_{\mathrm{L}}, \mathrm{U}(1)_{\mathrm{Y}}$, and $\mathrm{G}_{\mathrm{GUT}}$, respectively.2 are a more robust prediction of gauge unification. However, these simple relations (11) may also arise in models with no unified gauge group at high scales, as in some string theories with dilaton-dominated SUSY breaking [3], or theories with low energy dynamical SUSY breaking [4] [3 Thus, we conclude that if the sfermion masses do not satisfy the GUT mass relations, grand unification is by no means ruled out. On the other hand, verifying the gaugino mass relations, while extremely exciting, would not suffice as a proof for grand unification.

However, what if the opposite happened? Then there would be little question as to quarklepton unification, but what of gauge unification? In this letter, we point out that non-unified gaugino masses can in fact arise naturally in a very interesting class of grand unified theories, providing a unique signature for these type of models and a window into physics above the GUT scale.

Grand unified models suffer from a serious problem, the "doublet-triplet splitting problem". With a unified gauge group like $\mathrm{SU}(5)$ or $\mathrm{SO}(10)$, Higgs doublets are accompanied by color-triplet Higgses. Higgs doublets are responsible for the electro-weak symmetry breaking, and hence their masses are of the order of electro-weak scale. On the other hand, the

\footnotetext{
${ }^{1}$ We neglect the higher loop effects of $\mathrm{SU}(3)_{\mathrm{C}} \times \mathrm{SU}(2)_{\mathrm{L}} \times \mathrm{U}(1)_{\mathrm{Y}}$ gauge coupling constants, as they are quite small.

${ }^{2}$ We choose the GUT normalization for $g_{1}$, i.e., $g_{1}=\sqrt{\frac{5}{3}} g_{\mathrm{Y}}$.

${ }^{3}$ In the case of low energy dynamical SUSY breaking, the usual gaugino mass relations only follow if the vector-like fields transmitting SUSY breaking to the ordinary sector form complete representations of SU(5), which can perhaps be taken as indirect evidence for unification.
} 
stability of the nucleon and/or successful unification of the gauge coupling constants require the colored Higgs masses to be of the order of the GUT scale, $M_{\mathrm{GUT}} \sim 10^{16} \mathrm{GeV}$, which is much larger than the doublet Higgs masses [5]. In the minimal SUSY SU(5) model, this mass hierarchy is obtained by an extreme fine tune among several parameters in the superpotential. Many attempts have been made to solve this problem [6, 7, 8].

Recently, an interesting mechanism has been proposed to solve the doublet-triplet splitting problem [9, 10, 11, 12]. It is based on an enlarged gauge group, $\mathrm{G}_{\mathrm{GUT}} \times \mathrm{G}_{\mathrm{H}}$, like $\mathrm{SU}(5)_{\mathrm{GUT}} \times$ $\mathrm{SU}(3)_{\mathrm{H}}\left(\times \mathrm{U}(1)_{\mathrm{H}}\right)$ or $\mathrm{SO}(10)_{\mathrm{GUT}} \times \mathrm{SO}(6)_{\mathrm{H}}$ (where the subscript " $\mathrm{H}$ " stands for hypercolor). A characteristic feature of these models is that the $\mathrm{SU}(3)_{\mathrm{C}}$ group is a diagonal subgroup of $\mathrm{SU}(3)_{\text {GUT }}$ and $\mathrm{SU}(3)_{\mathrm{H}}$, where $\mathrm{SU}(3)_{\text {GUT }} \in \mathrm{G}_{\mathrm{GUT}}$ and $\mathrm{SU}(3)_{\mathrm{H}} \in \mathrm{G}_{\mathrm{H}}$, while $\mathrm{SU}(2)_{\mathrm{L}}$ is embedded only in $\mathrm{G}_{\mathrm{GUT}}$. The doublet-triplet splitting problem is solved by the missing partner mechanism [6], which in this case can work with smaller matter multiplet representations, guaranteeing a perturbative picture of $\mathrm{G}_{\mathrm{GUT}}$ up to the Planck scale.

Even though the unified gauge group is not simple, unification of the gauge coupling constants of $\mathrm{SU}(3)_{\mathrm{C}} \times \mathrm{SU}(2)_{\mathrm{L}} \times \mathrm{U}(1)_{\mathrm{Y}}$ is not spoiled if the gauge couplings of $\mathrm{G}_{\mathrm{H}}$ are large enough. In this case, the corrections to the gauge couplings of the low energy gauge group can be smaller than what can be distinguished by precision tests of the gauge coupling constants. However, we will show that the gaugino masses may deviate from the usual GUT relation (1), while scalar masses should still be unified at the GUT scale if they belong to the same multiplet of the GUT group.

Let us first review the $\mathrm{G}_{\mathrm{GUT}} \times \mathrm{G}_{\mathrm{H}}$ model. To make our points clear, we will concentrate on the model based on the gauge group $\mathrm{SU}(5)_{\mathrm{GUT}} \times \mathrm{SU}(3)_{\mathrm{H}} \times \mathrm{U}(1)_{\mathrm{H}}$ given in Ref. [10]. The generalization to other models is straightforward.

In the $\mathrm{SU}(5)_{\mathrm{GUT}} \times \mathrm{SU}(3)_{\mathrm{H}} \times \mathrm{U}(1)_{\mathrm{H}}$ model, the particle content which is responsible for the breaking of $\mathrm{G}_{\mathrm{GUT}} \times \mathrm{G}_{\mathrm{H}}$ group consists of the following chiral supermultiplets: $Q\left(\mathbf{5}^{*}, \mathbf{3}, \mathbf{1}\right)$, $\bar{Q}\left(\mathbf{5}, \mathbf{3}^{*},-1\right), q(\mathbf{1}, \mathbf{3}, 1), \bar{q}\left(\mathbf{1}, \mathbf{3}^{*},-1\right), \Sigma(\mathbf{2 4}, \mathbf{1}, 0), H(\mathbf{5}, \mathbf{1}, 0)$, and $\bar{H}\left(\mathbf{5}^{*}, \mathbf{1}, 0\right)$, where the numbers in brackets denote the transformation properties under $\mathrm{SU}(5)_{\mathrm{GUT}}, \mathrm{SU}(3)_{\mathrm{H}}$ and $\mathrm{U}(1)_{\mathrm{H}}$, respectively. The superpotential of the model is given by

$$
W=\bar{Q}_{A}^{\alpha}\left(m_{Q} \delta_{B}^{A}+\lambda \Sigma_{B}^{A}\right) Q_{\alpha}^{B}+\frac{1}{2} m_{\Sigma} \operatorname{tr}\left(\Sigma^{2}\right)+h H_{A} Q_{\alpha}^{A} \bar{q}^{\alpha}+h^{\prime} \bar{H}^{A} \bar{Q}_{A}^{\alpha} q_{\alpha},
$$

where $\lambda, h$ and $h^{\prime}$ are dimensionless coupling constants, while $m_{Q}$ and $m_{\Sigma}$ are mass parameters of the order of the GUT scale $M_{\mathrm{GUT}} \sim 10^{16} \mathrm{GeV}$. Minimizing the potential, we find that there is a vacuum in which the scalar components of $Q, \bar{Q}$, and $\Sigma$ have the following vacuum 
expectation values (VEVs),

$$
\begin{aligned}
& \langle Q\rangle=\left\langle\bar{Q}^{\mathrm{T}}\right\rangle=\left(\begin{array}{ccccc}
0 & 0 & v & 0 & 0 \\
0 & 0 & 0 & v & 0 \\
0 & 0 & 0 & 0 & v
\end{array}\right), \\
& \langle\Sigma\rangle=\frac{m_{Q}}{2 \lambda} \operatorname{diag}(3,3,-2,-2,-2),
\end{aligned}
$$

with $v^{2}=5 m_{Q} m_{\Sigma} / \lambda^{2}$, while other fields do not acquire a VEV.

In the vacuum given in eqs.(3) and (4), the gauge symmetry $\mathrm{SU}(5)_{\mathrm{GUT}} \times \mathrm{SU}(3)_{\mathrm{H}} \times \mathrm{U}(1)_{\mathrm{H}}$ is broken down to the standard model gauge group $\mathrm{SU}(3)_{\mathrm{C}} \times \mathrm{SU}(2)_{\mathrm{L}} \times \mathrm{U}(1)_{\mathrm{Y}}$ at the GUT scale. As we can see from the last two terms in eq.(2), the colored Higgses, $H_{I}$ and $\bar{H}^{I}(I$ $=3-5$ ), get large masses from the $Q, \bar{Q}$ VEVs by marrying with $q^{\alpha}$ and $\bar{q}_{\alpha}$, while doublet Higgses, $H_{i}$ and $\bar{H}^{i}(i=1,2)$, remain massless. Thus, the doublet-triplet splitting is naturally achieved in this model due to the missing partner mechanism.

An important feature of the $\mathrm{G}_{\mathrm{GUT}} \times \mathrm{G}_{\mathrm{H}}$ model is that the low energy $\mathrm{SU}(3)_{\mathrm{C}}$ symmetry is the diagonal subgroup of $\mathrm{SU}(3)_{\mathrm{GUT}} \times \mathrm{SU}(3)_{\mathrm{H}}$, where $\mathrm{SU}(3)_{\mathrm{GUT}}$ is embedded in $\mathrm{SU}(5)_{\text {GUT }}$. The gauge field of $\mathrm{SU}(3)_{\mathrm{C}}, G_{\mu}$, is given by a linear combination of the gauge field of $\mathrm{SU}(5)_{\mathrm{GUT}}$, $A_{\mathrm{GUT}, \mu}$, and of $\mathrm{SU}(3)_{\mathrm{H}}, A_{\mathrm{H} 3, \mu}$, as

$$
G_{\mu}=\frac{1}{\sqrt{g_{\mathrm{H} 3}^{2}+g_{\mathrm{GUT}}^{2}}}\left(g_{\mathrm{H} 3} A_{\mathrm{GUT}, \mu}+g_{\mathrm{GUT}} A_{\mathrm{H} 3, \mu}\right),
$$

where $g_{\mathrm{GUT}}$ and $g_{\mathrm{H} 3}$ are the gauge coupling constants for $\mathrm{SU}(5)_{\mathrm{GUT}}$ and $\mathrm{SU}(3)_{\mathrm{H}}$ at the GUT scale, and the gauge coupling $g_{3}$ of $\mathrm{SU}(3)_{\mathrm{C}}$ is given by, (in the following equations $(6)-(11)$, all relations are understood to hold at the GUT scale)

$$
g_{3}^{2}=\frac{g_{\mathrm{H} 3}^{2} g_{\mathrm{GUT}}^{2}}{g_{\mathrm{H} 3}^{2}+g_{\mathrm{GUT}}^{2}},
$$

or in terms of $\alpha\left(=g^{2} / 4 \pi\right)$,

$$
\frac{1}{\alpha_{3}}=\frac{1}{\alpha_{\mathrm{GUT}}}+\frac{1}{\alpha_{\mathrm{H} 3}}
$$

Similarly, $\mathrm{U}(1)_{\mathrm{Y}}$ is a subgroup of $\mathrm{U}(1)_{\mathrm{GUT}} \times \mathrm{U}(1)_{\mathrm{H}}$, and its gauge field $B_{\mu}$ and gauge coupling constant $g_{1}$ are given by

$$
\begin{aligned}
B_{\mu} & =\frac{1}{\sqrt{15 g_{\mathrm{H} 1}^{2}+g_{\mathrm{GUT}}^{2}}}\left(\sqrt{15} g_{\mathrm{H} 1} A_{\mathrm{GUT}, \mu}+g_{\mathrm{GUT}} A_{\mathrm{H} 1, \mu}\right), \\
g_{1}^{2} & =\frac{15 g_{\mathrm{H} 1}^{2} g_{\mathrm{GUT}}^{2}}{15 g_{\mathrm{H} 1}^{2}+g_{\mathrm{GUT}}^{2}} .
\end{aligned}
$$


On the other hand, $\mathrm{SU}(2)_{\mathrm{L}}$ is embedded only in $\mathrm{SU}(5)_{\mathrm{GUT}}$, and hence its gauge field $W_{\mu}$ and gauge coupling constant $g_{2}$ are given by

$$
\begin{aligned}
W_{\mu} & =A_{\mathrm{GUT}, \mu}, \\
g_{2}^{2} & =g_{\mathrm{GUT}}^{2} .
\end{aligned}
$$

The important point is that $g_{1}\left(M_{\mathrm{GUT}}\right) \simeq g_{2}\left(M_{\mathrm{GUT}}\right) \simeq g_{3}\left(M_{\mathrm{GUT}}\right)$ if $g_{\mathrm{H} 3}\left(M_{\mathrm{GUT}}\right), g_{\mathrm{H} 1}\left(M_{\mathrm{GUT}}\right)$ $\gg g_{\mathrm{GUT}}\left(M_{\mathrm{GUT}}\right)$. Thus, the gauge coupling unification is not spoiled if the gauge coupling constants of $\mathrm{G}_{\mathrm{H}}$ are large enough. As a result, it is very difficult to distinguish $\mathrm{G}_{\mathrm{GUT}} \times \mathrm{G}_{\mathrm{H}}$ model with the ordinary GUTs by the precise measurements of the gauge coupling constants. In fact, recent analyses show that the predicted strong coupling constant from SUSY-GUT without including threshold corrections, $\alpha_{s}\left(M_{Z}\right)=0.130$ [13, 14], is a little bit higher than the world averaged experimental value, $\alpha_{s}\left(M_{Z}\right)=0.118 \pm 0.003$ [15]. We can see from eqs.(6) and (7) that the correction from the hypercolor gauge coupling reduces the $\mathrm{SU}(3)_{\mathrm{C}}$ gauge coupling, and hence shifts the prediction in the right direction to be consistent with the experimental value. The correction from the $\mathrm{U}(1)_{\mathrm{H}}$ coupling also moves the prediction in the right direction by changing the unification scale. Let the shift of the inverse couplings of $\mathrm{SU}(3)_{\mathrm{C}}$ and $\mathrm{U}(1)_{\mathrm{Y}}$ at the GUT scale due to $\mathrm{G}_{\mathrm{H}}$ couplings be $\delta_{3}$ and $\delta_{1}$,

$$
\delta_{3}=\frac{1}{\alpha_{3}\left(M_{G}\right)}-\frac{1}{\alpha_{\mathrm{GUT}}\left(M_{G}\right)}=\frac{1}{\alpha_{3 \mathrm{H}}}, \quad \delta_{1}=\frac{1}{\alpha_{1}\left(M_{G}\right)}-\frac{1}{\alpha_{\mathrm{GUT}}\left(M_{G}\right)}=\frac{1}{15 \alpha_{1 \mathrm{H}}} .
$$

A simple calculation using one-loop renormalization group equations (RGEs) gives the shift of the inverse of the predicted strong coupling constant due to $\delta_{3}$ and $\delta_{1}$ as

$$
\Delta\left(\frac{1}{\alpha_{s}}\right)=\delta_{3}+\frac{5}{7} \delta_{1}
$$

So, the prediction of $\alpha_{s}\left(M_{Z}\right)$ in this model can be written as

$$
\alpha_{s}\left(M_{Z}\right) \simeq 0.130-\frac{0.014}{\alpha_{3 \mathrm{H}}}-\frac{0.010}{15 \alpha_{1 \mathrm{H}}}+\Delta_{\alpha_{s}}
$$

where the first term is the ordinary SUSY-GUT prediction, and the last term represents the extra threshold corrections. Typically it is found that $\left|\Delta_{\alpha_{s}}\right|<0.01$ [13]. Then in order to be consistent with the experimental value, we require $\alpha_{3 \mathrm{H}} \gtrsim 0.6$ (for $\delta_{1}=0$ ) and $\alpha_{1 \mathrm{H}} \gtrsim 0.03$ (for $\left.\delta_{3}=0\right)$.

Now, we are in a position to discuss the gaugino masses in the $\mathrm{G}_{\mathrm{GUT}} \times \mathrm{G}_{\mathrm{H}}$ model. The gaugino masses originate in the soft SUSY breaking terms, whose origin is related to the mechanism of the SUSY breaking. In the main part of this letter, we assume a hidden-sector 
SUSY breaking scenario, in which SUSY breaking is mediated by supergravity. The gauginos have the following mass terms above the GUT scale,

$$
\mathcal{L}=-\frac{1}{2} m_{\mathrm{GUT}} \lambda_{\mathrm{GUT}} \lambda_{\mathrm{GUT}}-\frac{1}{2} m_{\mathrm{H} 3} \lambda_{\mathrm{H} 3} \lambda_{\mathrm{H} 3}-\frac{1}{2} m_{\mathrm{H} 1} \lambda_{\mathrm{H} 1} \lambda_{\mathrm{H} 1}+\text { h.c. }
$$

where $\lambda_{\mathrm{GUT}}, \lambda_{\mathrm{H} 3}$ and $\lambda_{\mathrm{H} 1}\left(m_{\mathrm{GUT}}, m_{\mathrm{H} 3}\right.$ and $\left.m_{\mathrm{H} 1}\right)$ are gauginos (gaugino masses) for $\mathrm{SU}(5)_{\mathrm{GUT}}$, $\mathrm{SU}(3)_{\mathrm{H}}$ and $\mathrm{U}(1)_{\mathrm{H}}$ gauge groups, respectively. Heavy particles decouple at the GUT scale, and below the GUT scale, we have the SUSY standard model as a low energy effective theory. In particular, gauginos for the standard model gauge group $\mathrm{SU}(3)_{\mathrm{C}}, \mathrm{SU}(2)_{\mathrm{L}}$ and $\mathrm{U}(1)_{\mathrm{Y}}$ (which we denote $\tilde{G}, \tilde{W}$ and $\tilde{B}$ ), are given by, (eqs.(16) - (22) are understood to hold at the GUT scale)

$$
\begin{aligned}
\tilde{G} & =\frac{1}{\sqrt{g_{\mathrm{H} 3}^{2}+g_{\mathrm{GUT}}^{2}}}\left(g_{\mathrm{H} 3} \lambda_{\mathrm{GUT}}+g_{\mathrm{GUT}} \lambda_{\mathrm{H} 3}\right), \\
\tilde{W} & =\lambda_{\mathrm{GUT}}, \\
\tilde{B} & =\frac{1}{\sqrt{15 g_{\mathrm{H} 1}^{2}+g_{\mathrm{GUT}}^{2}}}\left(\sqrt{15} g_{\mathrm{H} 1} \lambda_{\mathrm{GUT}}+g_{\mathrm{GUT}} \lambda_{\mathrm{H} 1}\right) .
\end{aligned}
$$

Substituting eqs.(16) - (18) into eq. (15), we obtain masses for $\tilde{G}, \tilde{W}$ and $\tilde{B}$ as

$$
\begin{aligned}
& m_{3}=g_{3}^{2}\left(\frac{m_{\mathrm{H} 3}}{g_{\mathrm{H} 3}^{2}}+\frac{m_{\mathrm{GUT}}}{g_{\mathrm{GUT}}^{2}}\right), \\
& m_{2}=m_{\mathrm{GUT}}, \\
& m_{1}=g_{1}^{2}\left(\frac{m_{\mathrm{H} 1}}{15 g_{\mathrm{H} 1}^{2}}+\frac{m_{\mathrm{GUT}}}{g_{\mathrm{GUT}}^{2}}\right) .
\end{aligned}
$$

We can see that the GUT relation on the gaugino masses (1) is modified

$$
\frac{m_{3}}{g_{3}^{2}}: \frac{m_{2}}{g_{2}^{2}}: \frac{m_{1}}{g_{1}^{2}}=\left(\frac{m_{\mathrm{GUT}}}{g_{\mathrm{GUT}}^{2}}+\frac{m_{\mathrm{H} 3}}{g_{\mathrm{H} 3}^{2}}\right): \frac{m_{\mathrm{GUT}}}{g_{\mathrm{GUT}}^{2}}:\left(\frac{m_{\mathrm{GUT}}}{g_{\mathrm{GUT}}^{2}}+\frac{m_{\mathrm{H} 1}}{15 g_{\mathrm{H} 1}^{2}}\right) \text {. }
$$

The above relations receive negligible modification in running from the GUT to the weak scale. Thus, if the ratio $m_{\mathrm{H} 3} / g_{\mathrm{H} 3}^{2}$ or $m_{\mathrm{H} 1} / g_{\mathrm{H} 1}^{2}$ at the GUT scale is comparable to $m_{\mathrm{GUT}} / g_{\mathrm{GUT}}^{2}$, significant deviations from the usual GUT relations (1) can be observed when gaugino masses are measured. Notice that the combinations $m_{\mathrm{H} 3} / g_{\mathrm{H} 3}^{2}$ and $m_{\mathrm{H} 1} / g_{\mathrm{H} 1}^{2}$ are renormalization group invariants at the one loop level. So, in contrast with the gauge coupling, at one loop the corrections to the gaugino mass relations do not diminish as the hyper gauge couplings become large. On the other hand, in this model, squarks and sleptons are contained in $\left(\mathbf{5}^{*}, \mathbf{1}, 0\right)$ or $(\mathbf{1 0}, \mathbf{1}, 0)$ representation of the unified gauge group, as in the ordinary GUT. Thus, sfermion 
mass unification is still expected. These facts suggest that the mass spectroscopy of the superparticles can give us a signal for these kind of models.

Now, let us discuss the magnitude of $m_{\mathrm{H}} / g_{\mathrm{H}}^{2}$. In the hidden sector SUSY breaking scenario, gaugino masses are usually given in the form

$$
\mathcal{L}=\sum_{\mathrm{G}} \int d^{2} \theta \frac{k_{\mathrm{G}}}{M_{\mathrm{PL}}} S W^{\mathrm{G}} W^{\mathrm{G}}+\text { h.c. },
$$

where $S$ denotes the chiral multiplet which is responsible for the SUSY breaking, $M_{\mathrm{PL}} \simeq$ $2.4 \times 10^{18} \mathrm{GeV}$ is the reduced Planck scale, $k_{\mathrm{G}}$ denote the coupling constants, and $W^{\mathrm{G}}$ is the superfield for the gauge multiplet. (Here, G indicates gauge group.) Then, when SUSY is broken $\left(F_{S} \equiv\left\langle\int d^{2} \theta S\right\rangle \neq 0\right)$, we will get gaugino masses of order $k_{\mathrm{G}} F_{S} / M_{\mathrm{PL}}$. In general, we do not expect that $k_{\mathrm{H} 3}$ and $k_{\mathrm{H} 1}$ are much smaller than $k_{\mathrm{GUT}}$, and hence the deviation from the GUT relation is expected to be non-negligible. To make a more definite statement we have to make assumptions about how SUSY is broken. For example, in the superstring inspired model with dilaton-dominated SUSY breaking [3], the combination $m_{\mathrm{G}} / g_{\mathrm{G}}^{2}$ is universal for all the gauge groups $\mathrm{G}$ at the string scale. Then, large corrections to the GUT gaugino mass relations are expected. In particular, the gaugino masses for $\mathrm{SU}(3)_{\mathrm{C}}$ and $\mathrm{SU}(2)_{\mathrm{L}}$ obey $m_{3} / g_{3}^{2}: m_{2} / g_{2}^{2}=2: 1$, neglecting the higher order corrections.t. In general, the ratio,

$$
R_{3 / 2} \equiv \frac{m_{3} / g_{3}^{2}}{m_{2} / g_{2}^{2}}
$$

is related to the boundary condition,

$$
\left.R_{\mathrm{H} / \mathrm{G}} \equiv \frac{m_{\mathrm{H} 3} / g_{\mathrm{H} 3}^{2}}{m_{\mathrm{GUT}} / g_{\mathrm{GUT}}^{2}}\right|_{\mu=M_{\mathrm{PL}}},
$$

as $R_{3 / 2}=R_{\mathrm{H} / \mathrm{G}}+1$ in the one loop approximation (with $\mu$ being the renormalization point).

So far, we considered only one loop RGEs. However, as we discussed before, gauge coupling constants for $\mathrm{G}_{\mathrm{H}}$ have to be large at the GUT scale, and hence the results based on the one loop RGEs may not be a good approximation. In fact, the ratio of the gaugino mass to the gauge coupling constant squared receives higher order corrections and does not remain constant. Therefore, $R_{3 / 2}$ will depend explicitly on $g_{\mathrm{H} 3}\left(M_{G}\right)$ as well as on $R_{\mathrm{H} / \mathrm{G}}$ if we take into account the higher loop effects due to the large coupling $g_{\mathrm{H} 3}$.

In order to demonstrate the effect of the higher order terms, we use two loop RGEs to evolve the ratios between the GUT scale and the Planck scale. The two loop RGEs for gauge

\footnotetext{
${ }^{4}$ For $\mathrm{U}(1)_{\mathrm{H}}$, we do not know the normalization of the charges of the chiral multiplets, and hence we cannot give definite prediction on $\mathrm{U}(1)_{\mathrm{Y}}$.
} 
couplings and gaugino masses are given in [16]. We fix $\alpha_{\mathrm{GUT}}\left(=g_{\mathrm{GUT}}^{2} / 4 \pi\right)=1 / 25$ at the GUT scale, and numerically evaluate $R_{3 / 2}$ as a function of $\alpha_{\mathrm{H} 3}\left(M_{\mathrm{GUT}}\right)$ for different initial values of $R_{\mathrm{H} / \mathrm{G}}$ at $M_{\mathrm{PL}}$. The result is shown in Fig. 1 for $0.5<\alpha_{\mathrm{H} 3}\left(M_{\mathrm{GUT}}\right)<2$. We can see that $R_{3 / 2}$ is close to the one loop value, $R_{\mathrm{H} / \mathrm{G}}+1$, for smaller $\alpha_{\mathrm{H} 3}$ and $R_{\mathrm{H} / \mathrm{G}}$, but deviates significantly from the one loop result for larger $\alpha_{\mathrm{H} 3}$ and $R_{\mathrm{H} / \mathrm{G}}$. Notice that the apparent blow up of $R_{3 / 2}$ in the case with $R_{\mathrm{H} / \mathrm{G}}=2$ is due to $m_{\mathrm{GUT}}$ being scaled to zero in the course of running. Below $M_{\mathrm{GUT}}, R_{3 / 2}$ stays approximately constant since there is no large coupling to make higher loop contributions important. For very large $\alpha_{\mathrm{H} 3}$, the perturbative calculation should break down and the results based on the two loop calculation are not reliable. In that case, we have no control on $m_{\mathrm{H} 3} / g_{\mathrm{H} 3}^{2}$ near the GUT scale. However, based on our results for moderate large $\alpha_{\mathrm{H} 3}$, we do not expect that $m_{\mathrm{H} 3} / g_{\mathrm{H} 3}^{2}$ quickly goes to zero for finite values of $\alpha_{\mathrm{H} 3}$.

Some comments are in order. First of all, we would like to discuss the models based on the gauge group other than $\mathrm{SU}(5)_{\mathrm{GUT}} \times \mathrm{SU}(3)_{\mathrm{H}} \times \mathrm{U}(1)_{\mathrm{H}}$, i.e. models based on $\mathrm{SU}(5)_{\mathrm{GUT}} \times$ $\mathrm{SU}(3)_{\mathrm{H}}$ [11] or $\mathrm{SO}(10)_{\mathrm{GUT}} \times \mathrm{SO}(6)_{\mathrm{H}}$ [12]. In those cases, $\mathrm{SU}(3)_{\mathrm{C}}$ is a diagonal subgroup of $\mathrm{SU}(3)_{\mathrm{GUT}}$ and $\mathrm{SU}(3)_{\mathrm{H}}$ as in the previous case, while $\mathrm{SU}(2)_{\mathrm{L}}$ and $\mathrm{U}(1)_{\mathrm{Y}}$ are embedded only in $\mathrm{G}_{\mathrm{GUT}}$. Then, the gaugino masses obey the relation (22) with $m_{\mathrm{H} 1}=0$, i.e., the gaugino masses for $\mathrm{SU}(2)_{\mathrm{L}}$ and $\mathrm{U}(1)_{\mathrm{Y}}$ obey the usual GUT relation, while that for $\mathrm{SU}(3)_{\mathrm{C}}$ does not. This kind of signal, together with the unifications of the sfermion masses, will give us an information on the structure of the $\mathrm{G}_{\mathrm{GUT}} \times \mathrm{G}_{\mathrm{H}}$ model.

So far, we have concentrated on the hidden sector SUSY breaking scenario. There is another interesting scenario where supersymmetry is broken dynamically at a low energy scale, then mediated to the observable sector by a messenger sector [4]. To preserve gauge coupling unification, the messenger sector should fill out complete multiplets under ordinary $\mathrm{SU}(5)$. In this case, the GUT relation (1) is not affected even in the $\mathrm{G}_{\mathrm{GUT}} \times \mathrm{G}_{\mathrm{H}}$ model.

In summary, we have investigated the gaugino masses in supersymmetric unified models based on an enlarged gauge group like $\mathrm{SU}(5)_{\mathrm{GUT}} \times \mathrm{SU}(3)_{\mathrm{H}}\left(\times \mathrm{U}(1)_{\mathrm{H}}\right)$ or $\mathrm{SO}(10)_{\mathrm{GUT}} \times \mathrm{SO}(6)_{\mathrm{H}}$, in which the doublet-triplet splitting problem can be solved naturally. In these models, the GUT relations on the gaugino masses can be broken completely or partially, while we can still hope that the unification of the sfermion masses is unaffected. Therefore, by the accurate spectroscopy of the superparticles, we may have a window into physics at and beyond the GUT scale.

The authors would like to thank L.J. Hall and H. Murayama for useful discussions. This work was supported in part by the Director, Office of Energy Research, Office of High Energy and Nuclear Physics, Division of High Energy Physics of the U.S. Department of Energy under Contract DE-AC03-76SF00098 and in part by the National Science Foundation under 
grant PHY-95-14797, and the work of N.A.-H. is supported by NSERC.

\section{References}

[1] S. Dimopoulos and H. Georgi, Nucl. Phys. B193 (1981) 150;

N. Sakai, Z. Phys. C11 (1981) 153.

[2] P. Langacker and M. Luo, Phys. Rev. D44 (1991) 817;

U. Amaldi, W. de Boer and H. Fürstenau, Phys. Lett. B260 (1991) 447.

[3] V.S. Kaplunovsky and J. Louis, Phys. Lett. B306 (1993) 269;

A. Brignole, L.E. Ibáñez and C. Muñoz, Nucl. Phys. B422 (1994) 125.

[4] M. Dine, A.E. Nelson and Y. Shirman, Phys. Rev. D51 (1995) 1362;

M. Dine, A.E. Nelson, Y. Nir and Y. Shirman, Phys. Rev. D53 (1996) 2658.

[5] J. Hisano, H. Murayama and T. Yanagida, Nucl. Phys. B402 (1993) 46;

J. Hisano, T. Moroi, K. Tobe and T. Yanagida, Mod. Phys. Lett. A10 (1995) 2267.

[6] A. Masiero, D.V. Nanopoulos, K. Tamvakis and T. Yanagida, Phys. Lett. B115 (1982) 380 ;

B. Grinstein, Nucl. Phys. B206 (1982) 387.

[7] S. Dimopoulos and F. Wilczek, Proceedings Erice Summer School, Ed. A. Zichichi, 1981; K.S. Babu and S.M. Barr, Phys. Rev. D48 (1993) 5354; Phys. Rev. D50 (1994) 3529;

J. Hisano, H. Murayama and T. Yanagida, Phys. Rev. D49 (1994) 4966.

[8] K. Inoue, A. Kakuto and T. Takano, Prog. Theor. Phys. 75 (1986) 664;

A. Anselm and A. Johansen, Phys. Lett. B200 (1988) 331;

Z. Berezhiani and G. Dvali, Sov. Phys. Lebedev Inst. Rep. 5 (1989) 55;

R. Barbieri, G. Dvali and M. Moretti, Phys. Lett. B312 (1993) 137;

Z. Berezhiani C. Csaki and L. Randall, Nucl. Phys. B441 (1995) 61.

[9] T. Yanagida, Phys. Lett. B344 (1995) 211.

[10] T. Hotta, K.-I. Izawa and T. Yanagida, Phys. Rev. D53 (1996) 3913.

[11] T. Hotta, K.-I. Izawa and T. Yanagida, preprint UT-733 (hep-ph/9511431).

[12] T. Hotta, K.-I. Izawa and T. Yanagida, preprint UT-743 (hep-ph/9602439). 
[13] P. Langacker and N. Polonsky, Phys. Rev. D52 (1995) 3081.

[14] J. Bagger, K. Matchev and D. Pierce, Phys. Lett. B348 (1995) 443;

J. Bagger, K. Matchev, D. Pierce and R. Zhang, preprint SLAC-PUB-7180 hepph/9606211).

[15] Review of Particle Properties, R.M. Barnett et al., Phys. Rev. D54 (1996) 1.

[16] Y. Yamada, Phys. Rev. Lett. 72 (1994) 25;

S.P. Martin and M.T. Vaughn, Phys. Rev. D50 (1994) 2282;

I. Jack and D.R.T. Jones, Phys. Lett. B333 (1994) 372. 


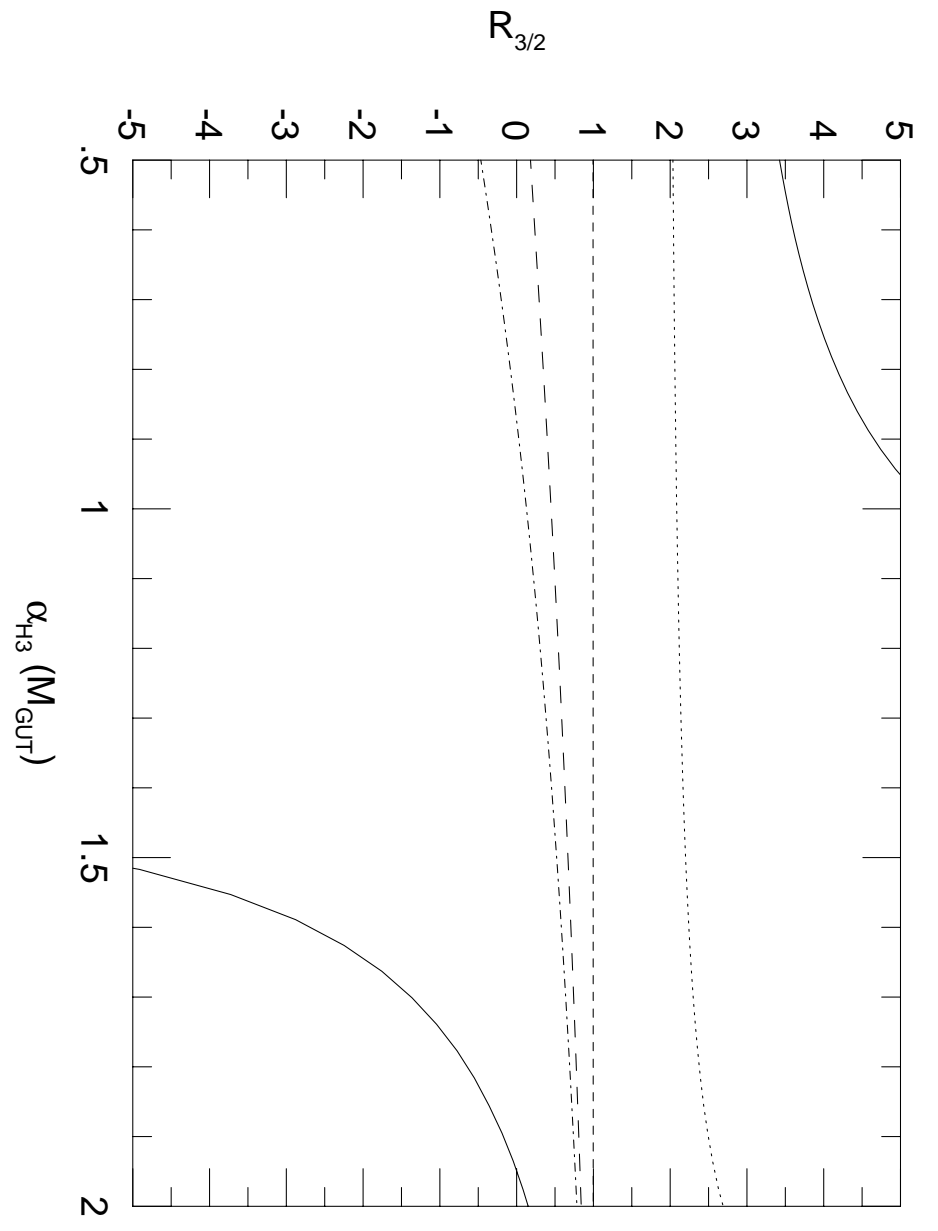

Figure 1: The ratio $R_{3 / 2}$ as a function of $\alpha_{\mathrm{H} 3}\left(M_{\mathrm{GUT}}\right)$ for different values of the ratio $\mathrm{R}_{\mathrm{H} / \mathrm{G}}=2$ (solid), 1 (dotted), 0 (short dashed), -1 (long dashed) and -2 (dot-dashed). 